\title{
Prevalencia al nacimiento de hipotiroidismo congénito
}

\section{Congenital hypothyroidism birth prevalence:}

Mirna Angélica Hinojosa-Trejo, ${ }^{1}$ Marcela Vela-Amieva, ${ }^{2}$ Isabel Ibarra-González, ${ }^{3}$ Ana Paola de Cosío-Farias, ${ }^{4}$ Luz del Alba Herrera-Pérez, ${ }^{5}$ Guillermo Caamal-Parra, ${ }^{5}$ Lidia Elizabeth Bolaños-Córdova, ${ }^{1}$ Erika Paola García-Flores ${ }^{1}$

\section{Resumen}

INTRODUCCIÓN: El hipotiroidismo congénito es la causa más común de discapacidad intelectual susceptible de prevención. En México, el tamiz neonatal de esta enfermedad es obligatorio. La prevalencia al nacimiento de hipotiroidismo congénito fue descrita en México, hace más de 10 años.

OBJETIVO: Presentar información actualizada de la prevalencia de hipotiroidismo congénito del Programa de Tamiz Neonatal de la Secretaría de Salud y compararla con la reportada previamente en el país.

MATERIALES Y MÉTODOS: Estudio retrospectivo de recién nacidos tamizados en las unidades médicas de la Secretaría de Salud del 1 de enero de 2017 al 31 de mayo de 2018. Se cuantificó la hormona estimulante de la tiroides (TSH) en sangre neonatal. Se revisó la bibliografía de la prevalencia al nacimiento de hipotiroidismo congénito (publicaciones del 2000 a la fecha).

RESULTADOS: En 1,267,122 muestras adecuadas para el procesamiento bioquímico se encontraron 3,337 con TSH $>10 \mu \mathrm{U} / \mathrm{mL}$. Se confirmaron 923 casos, de los que $564(61.1 \%)$ fueron niñas. La prevalencia al nacimiento fue de 7.3 por 10,000 recién nacidos. En la revisión bibliográfica solo se encontraron dos estudios con datos nacionales desglosados por entidad federativa, con una prevalencia de 4.1 y 4.3 x 10,000 recién nacidos.

CONCLUSIÓN: La prevalencia al nacimiento de hipotiroidismo congénito en los recién nacidos de la Secretaría de Salud de México fue de 7.3 x cada 10,000 recién nacidos tamizados, lo que significa que uno de cada 1,373 tiene esta condición; las niñas son las más afectadas. El hipotiroidismo congénito aumentó 1.8 veces en los últimos 14 años.

PALABRAS CLAVE: Tamiz metabólico; hipotiroidismo congénito; hormona estimulante de la tiroides.

\section{Abstract}

INTRODUCTION: Congenital hypothyroidism is the most common preventable cause of intellectual disability. In Mexico newborn screening (NBS) of this disease is mandatory. Congenital hypothyroidism birth prevalence has been previously described in Mexico, however, these studies date from more than 10 years ago.

OBJECTIVE: To present updated information on the congenital hypothyroidism birth prevalence from the NBS Program of the Ministry of Health and to compare it with those previously reported in Mexico.

MATERIALS AND METHOD: Retrospective study of newborns screened in the medical units of the Ministry of Health from January 1, 2017 to May 31, 2018. Thyroid stimulating hormone (TSH) was quantified in neonatal blood. A review from the literature was made searching the congenital hypothyroidism birth prevalence, considering the publications of the year 2000 to date.
${ }^{1}$ Centro Nacional de Equidad de Género y Salud Reproductiva, Secretaría de Salud, México.

${ }^{2}$ Laboratorio de Errores Innatos del Metabolismo y Tamiz, Instituto Nacional de Pediatría, Secretaría de Salud, México. ${ }^{3}$ Unidad de Genética de la Nutrición, Instituto de Investigaciones Biomédicas, UNAM, Instituto Nacional de Pediatría SS, México.

${ }^{4}$ Servicio de Endocrinología, Instituto Nacional de Pediatría, Secretaría de Salud, México.

${ }^{5}$ TamizMas Screening Center de Químicos Maldonado, Yucatán, México.

Correspondencia

Erika Paola García Flores

erika.garcia@salud.gob.mx

Este artículo debe citarse como Hinojosa Trejo MA, Vela Amieva M, IbarraGonzález I, Cosío Farias AP, Herrera Pérez LA, Caamal Parra G, Bolaños Córdova LE, García Flores EP. Prevalencia al nacimiento de hipotiroidismo congénito. Acta Pediatr Mex. 2018;SI(39):5S-13S 
RESULTS: In 1,267,122 samples suitable for biochemical analysis, we found 3.337 newborns with TSH > $10 \mu / \mathrm{mL} .923$ cases were confirmed and from them $564(61.1 \%)$ were girls. The congenital hypothyroidism birth prevalence was 7.3 per 10,000 screened newborns. From the bibliographic review, only two studies were found with national data disaggregated by federative entity, with a national prevalence of 4.1 and $4.3 \times 10,000$ newborns.

CONCLUSION: Congenital hypothyroidism birth prevalence in Mexico was $7.3 \times$ each 10,000 newborns screened in the Ministry of Health, which means that one in 1373 has this condition being, the girls the most affected ones. $\mathrm{CH}$ birth prevalence in has increased 1.8 times in the last 14 years.

KEYWORDS: Newborn screening, congenital hypothyroidism, thyroid stimulating hormone.

\section{INTRODUCCIÓN}

El tamiz neonatal metabólico inició en México en 1973 con un primer piloto que incluía la detección de fenilcetonuria, homocistinuria, enfermedad de jarabe de maple y tirosinemia, el cual fue cancelado a pesar de haber demostrado su factibilidad; en 1976 cuando se introdujo la detección de hipotiroidismo congénito y en 1986 se estableció un programa que inició en la Ciudad de México y se fue extendiendo a todo el país, el cual contempló la detección de hipotiroidismo congénito y fenilcetonuria. ${ }^{1}$ En 1996 se emitió en el Diario Oficial de la Federación la Norma Técnica No 321 para la prevención del retraso mental producido por hipotiroidismo congénito, con lo que se hizo obligatoria la realización del tamiz neonatal en todo el Sector Salud, en 1995 dicha norma se convierte en la Norma Oficial Mexicana NOM-007-SSA2-1993, Atención de la mujer durante el embarazo, parto y puerperio y del recién nacido. Criterios y procedimientos para la prestación del servicio, la cual hace obligatoria la determinación de esta enfermedad en todos los recién nacidos mexicanos. ${ }^{2,3}$ En el año 1996 se realiza la primera compra de insumos a nivel nacional para hipotiroidismo congénito, gracias a la inclusión de esta enfermedad al Presupuesto de Egresos de la Federación. ${ }^{4}$ En este mismo año, se dio un incremento significativo en la cobertura, pasando de 5.4\% que se tenía en 1989 a $87.9 \%$ en 1997, ocasionado por el cambio en el método de toma de muestra, al introducir la toma de cordón umbilical. ${ }^{5}$

Como resultado de un trabajo coordinado sectorial y multidisciplinario y con la participación de especialistas de todo el país, el Centro Nacional de Equidad de Género y Salud Reproductiva (CNEGSR) en el año 2007 emitió el primer Lineamiento Técnico: Tamiz neonatal: Detección y tratamiento oportuno e integral del hipotiroidismo congénito, ${ }^{6}$ el cuál es el detonador de una serie de actividades de capacitación, sensibilización, atención y seguimiento, dirigido a todos los niveles de atención que estaban involucrados en la atención de recién nacidos con problemas de discapacidad, así como a los padres a quienes se les da el apoyo e información para lograr la comprensión del padecimiento y la adherencia al tratamiento. A partir del año 2016, con la finalidad de mejorar los indicadores del programa y derivado del cambio en las reglas de operación del Seguro Médico Siglo XXI (fuente de financiamiento del programa), ${ }^{7}$ se realiza la contratación de servicios especializados e integrales que incluyen la dotación de insumos (papel filtro y lancetas), el análisis de muestras, la realización de pruebas confirmatorias y el sis- 
tema de información que permitiera la consulta en línea de los resultados, así como la impresión de los mismos.

El hipotiroidismo congénito se define como la deficiencia de hormonas tiroideas presente al nacimiento y es la causa más frecuente de patología endocrinológica en el recién nacido y la primera causa de retraso mental prevenible. Afecta con mayor frecuencia a pacientes femeninos con una relación 2:1 y 90\% de los casos son permanentes. ${ }^{8-12}$ Por lo general no presenta signos y síntomas notorios desde el nacimiento, por lo que es muy importante detectarlo tempranamente mediante el tamiz neonatal, puesto que el pronóstico neurológico depende del inicio oportuno y correcto del tratamiento. ${ }^{13}$ Los signos más prevalentes son ictericia y hernia umbilical. La ictericia suele ser de predominio indirecto. ${ }^{14}$ En el Cuadro 1 se resumen las principales características clínicas de la enfermedad. El hipotiroidismo congénito se asocia a un mayor riesgo de malformaciones a nivel renal, cardiaco y del sistema urinario. ${ }^{10}$

El tratamiento de elección del hipotiroidismo congénito es levotiroxina (LT4) y debe iniciarse de manera de manera inmediata. La dosis inicial es de 10 a $15 \mu \mathrm{g} / \mathrm{kg}$, los padres deben ser instruidos sobre la forma de dar el medicamento. 6,10,14

Cuadro 1. Principales características clínicas del hipotiroidismo congénito

- Ictericia

- Hernia umbilical

- Fontanela anterior amplia

- Sutura sagital ensanchada

- Hipotonía

- Hipotermia

- Macrosomía

- Succión débil

- Retraso en el desarrollo

- Macroglosia

- Llanto ronco

- Estreñimiento
La primera evaluación deberá ser una o dos semanas después del inicio del tratamiento con un perfil tiroideo. Se deberá monitorizar con pruebas de función tiroidea cada 2 semanas hasta Ilegar a valores normales de TSH para la edad, posteriormente cada 1 o 3 meses durante el primer año de vida. De los 2 a los 3 años cada 2 a 4 meses y posteriormente cada 3 a 12 meses. ${ }^{10,14}$

Antes de la implementación de los programas de tamiz neonatal en el mundo, la frecuencia de hipotiroidismo congénito era cercana a 1 en 7,000 nacidos vivos. ${ }^{9}$ En la actualidad se sabe que la prevalencia al nacimiento varía dependiendo de diversos factores tales como el área geográfica, la suficiencia de yodo en la población, la metodología y punto de corte utilizados para el análisis bioquímico. ${ }^{13,15}$ Diversos estudios han demostrado las variaciones étnicas en la frecuencia del hipotiroidismo congénito, siendo las poblaciones denominadas "hispánicas" (entre las que se consideran los mexicanos que residen en Estados Unidos), ${ }^{16}$ una de las más afectadas por la enfermedad, con cifras cercanas a $6.2 \times$ $10,000 .{ }^{17}$ En México la prevalencia al nacimiento de hipotiroidismo congénito ha sido previamente descrita, sin embargo, dichos estudios datan de hace más de 10 años. ${ }^{18,19}$

\section{OBJETIVO}

El objetivo de este trabajo es presentar información actualizada sobre la prevalencia al nacimiento de hipotiroidismo congénito del programa de tamiz neonatal de la Secretaría de Salud durante el periodo del 1 de enero de 2017 al 31 de mayo de 2018 y compararla con las previamente reportadas en México.

\section{MATERIALES Y MÉTODOS}

La población de estudio comprendió a 1,273,727 recién nacidos tamizados en el periodo del 1 de enero del 2017 al 31 de mayo del 2018, en las 
unidades médicas de la Secretaría de Salud a lo largo del territorio nacional, siguiendo la normatividad vigente. ${ }^{3,20}$ A todos los recién nacidos se les tomaron muestras de sangre capilar mediante el uso de lancetas especiales para tamiz neonatal y la sangre fue depositada en tarjetas de papel filtro de algodón (tarjeta de Guthrie). Las muestras se dejaron secar a temperatura ambiente por un mínimo de 3 horas y fueron enviadas por mensajería al laboratorio procesador, en dónde dos observadores revisaron su calidad (sangre suficiente en el papel filtro, ausencia de halos de suero, ausencia de coágulos y de contaminación, sobreposición de gotas, entre otras). Las muestras fueron perforadas en círculos de $3.2 \mathrm{~mm}$ de diámetro mediante instrumentos automatizados (Panthera-puncher-9, PerkinElmer ${ }^{\circledR}$ ). La hormona estimulante de la tiroides o tirotropina (TSH) fue cuantificada en la sangre neonatal utilizando estuches comerciales GSP ${ }^{\circledR}$ Neonatal hTSH (PerkinElmer-Wallac Oy, Mustionkatu 6, FI-20750 Turku, Finlandia). El análisis se realizó en el instrumento Genetic Screening Processor ${ }^{\circledR}$ de la marca Perkin Elmer ${ }^{\circledR}$, que determina los niveles de TSH mediante un ensayo en fase sólida fluoroinmunométrico de dos sitios. En cada placa de 96 pozos se emplearon seis calibradores por duplicado y 3 controles de concentraciones baja, media y alta. El laboratorio procesador de las muestras está inscrito al "Programa de control externo de la calidad" de los CDC (Centers for Disease Control and Prevention) de Atlanta y al "Programa de Evaluación Externa de la Calidad" de la Fundación Bioquímica Argentina. El punto de corte de corte utilizado fue de $10 \mu \mathrm{U} / \mathrm{mL}$ de sangre, de tal manera que todas las muestras con resultados por arriba de ese valor fueron analizadas nuevamente por duplicado. Las muestras cuyo resultado después del reproceso era consistentemente elevado, fueron reportadas como sospechosas a los responsables estatales del programa, los que se dieron a la tarea de buscar a los recién nacidos sospechosos para la realización de la prueba confirmatoria (perfil tiroideo). Los recién nacidos con resultado positivo en la prueba confirmatoria, fueron enviados a los hospitales de referencia estatal más cercanos a su domicilio para dar inicio a su tratamiento.

Se realizó una búsqueda y revisión de la literatura, incluyendo estudios publicados nacionales o internacionales sobre la prevalencia al nacimiento de hipotiroidismo congénito y considerando las publicaciones del año 2000 a la fecha con los términos "congenital hypothyroidism" [MeSH Terms] [All Fields] AND "birth prevalence" [MeSH Terms] OR "incidence" [MeSH Terms] AND "humans" [MeSH Terms] AND "Mexico" [MeSH Terms].

\section{RESULTADOS}

En el periodo de estudio se tamizaron 1,273,727 recién nacidos, de los cuales 1,267,122 muestras $(99.5 \%)$ fueron adecuadas para el procesamiento bioquímico. Se encontraron 3,337 muestras cuya concentración sanguínea de TSH fue mayor de $10 \mu \mathrm{U} / \mathrm{mL}$ y fueron notificadas de manera inmediata. Se confirmaron 923 casos, de los cuales $564(61.1 \%)$ fueron niñas, 357 (38.7\%) niños y en $2(0.2 \%)$ casos el sexo no se especificó. En relación con la edad gestacional, 853 (92.4\%) fueron de término, $62(6.7 \%)$ prematuros y 5 $(0.5 \%)$ postérmino y $3(0.3 \%)$ sin dato.

Se encontró una prevalencia al nacimiento de 7.3 por 10,000 recién nacidos vivos ( 1 caso de cada 1,373 recién nacidos vivos). La máxima frecuencia se observó en el estado de Durango (18.0 x 10,000 recién nacidos vivos) y la mínima se encontró en Tabasco (1.6 x 10,000 recién nacidos vivos). Los resultados desglosados por entidad federativa se muestran en el Cuadro 2.

De la revisión bibliográfica, se encontraron 40 referencias de las cuales, solo 2 presentan datos nacionales desglosados por entidad federativa. 
Hinojosa-Trejo MA, et al. Hipotiroidismo congénito

Cuadro 2. Recién nacidos tamizados, número de muestras adecuadas, casos sospechosos, confirmados y prevalencia al nacimiento de hipotiroidismo congénito en México

\begin{tabular}{|c|c|c|c|c|c|c|c|c|}
\hline Entidad & $\begin{array}{c}\text { RN } \\
\text { tamizados }\end{array}$ & $\begin{array}{c}\text { Muestras } \\
\text { adecuadas }\end{array}$ & $\begin{array}{c}\text { Muestras } \\
\text { sospechosas }\end{array}$ & Casos & $\begin{array}{c}\text { Prevalencia } \\
\text { estimada al } \\
\text { nacimiento } \\
(1: \mathrm{RN})\end{array}$ & $\begin{array}{l}\text { Rendón- } \\
\text { Macías } \\
2018\end{array}$ & $\begin{array}{c}\text { Vela- } \\
\text { Amieva } \\
2004\end{array}$ & $\begin{array}{c}\text { CNEGSR } \\
\text { (presente } \\
\text { trabajo) }\end{array}$ \\
\hline Aguascalientes & 16,896 & 16,878 & 39 & 14 & 1,206 & 2.7 & 3.3 & 8.3 \\
\hline Baja California Norte & 24,341 & 24,290 & 79 & 19 & 1,278 & 3.7 & 2.6 & 7.8 \\
\hline Baja California Sur & 6,272 & 6,245 & 10 & 4 & 1,561 & 5.1 & 7.5 & 6.4 \\
\hline Campeche & 11,683 & 11,646 & 23 & 14 & 832 & 3.3 & 6.5 & 12.0 \\
\hline Chiapas & 59,709 & 59,204 & 123 & 37 & 1,600 & 1.9 & 2.6 & 6.2 \\
\hline Chuhuahua & 25,699 & 25,593 & 49 & 12 & 2,133 & 2.9 & 3.2 & 4.7 \\
\hline Ciudad de México & 42,795 & 42,694 & 95 & 28 & 1,525 & 3.8 & 5.9 & 6.6 \\
\hline Coahuila & 22,634 & 22,465 & 72 & 18 & 1,248 & 3.8 & 3 & 8.0 \\
\hline Colima & 7,657 & 7,647 & 127 & 5 & 1,529 & 1.8 & 5.4 & 6.5 \\
\hline Durango & 20,705 & 20,583 & 74 & 37 & 556 & 2.9 & 2.3 & 18.0 \\
\hline Estado de México & 150,948 & 150,558 & 285 & 60 & 2,509 & 5.3 & 4.2 & 4.0 \\
\hline Guanajuato & 101,602 & 100,935 & 175 & 79 & 1,278 & 3.1 & 2.8 & 7.8 \\
\hline Guerrero & 61,337 & 61,212 & 144 & 82 & 756 & 3.9 & 3.4 & 13.4 \\
\hline Hidalgo & 39,430 & 39,385 & 75 & 17 & 2,317 & 4.3 & 6.3 & 4.3 \\
\hline Jalisco & 77,353 & 77,051 & 233 & 71 & 1,085 & 3.5 & 2.6 & 9.2 \\
\hline Michoacán & 64,516 & 64,114 & 161 & 45 & 1,425 & 5 & 5.1 & 7.0 \\
\hline Morelos & 22,739 & 22,727 & 40 & 19 & 1,196 & 4.3 & 3.5 & 8.4 \\
\hline Nayarit & 9,981 & 9,940 & 48 & 10 & 994 & 1.9 & 4.8 & 10.1 \\
\hline Nuevo León & 31,997 & 31,928 & 555 & 33 & 968 & 7.8 & 4.3 & 10.3 \\
\hline Oaxaca & 48,151 & 47,848 & 96 & 27 & 1,172 & 7 & 6.6 & 5.6 \\
\hline Puebla & 96,041 & 95,773 & 187 & 79 & 1,212 & 5.2 & 5.3 & 8.2 \\
\hline Querétaro & 24,351 & 23,951 & 33 & 17 & 1,409 & 4.2 & 3.4 & 7.1 \\
\hline Quintana Roo & 19,930 & 19,772 & 32 & 8 & 2,472 & 3 & 8.4 & 4.0 \\
\hline San Luis Potosí & 28,759 & 28,644 & 45 & 24 & 1,194 & 5.9 & 7.8 & 8.4 \\
\hline Sinaloa & 26,928 & 26,908 & 80 & 17 & 1,583 & 3.7 & 0.6 & 6.3 \\
\hline Sonora & 21,318 & 21,273 & 94 & 23 & 925 & 3.5 & 2.4 & 10.8 \\
\hline Tabasco & 31,556 & 31,235 & 62 & 5 & 6,247 & 4.7 & 1.6 & 1.6 \\
\hline Tamaulipas & 35,588 & 35,484 & 58 & 34 & 1,044 & 6.4 & 5 & 9.6 \\
\hline Tlaxcala & 19,524 & 19,506 & 41 & 6 & 3,251 & 2.9 & 5.3 & 3.1 \\
\hline Veracruz & 80,306 & 78,739 & 108 & 50 & 1,575 & 3.7 & 2.1 & 6.4 \\
\hline Yucatán & 22,715 & 22,715 & 40 & 22 & 1,033 & 4.6 & 0 & 9.7 \\
\hline Zacatecas & 20,66 & 20,179 & 54 & 7 & 2,883 & 3.3 & 4.1 & 3.5 \\
\hline Total & $1,273,727$ & $1,267,122$ & 3,337 & 923 & 1,373 & 4.3 & 4.1 & 7.3 \\
\hline
\end{tabular}


Los principales resultados y su comparación con el trabajo actual se muestran en el Cuadro 3. En la Figura 1 se muestran las prevalencias obtenidas, observándose que en la mayoría de los estados de la república han aumentado. En los estados de Tabasco, Tlaxcala, Zacatecas, Quintana Roo, Hidalgo, y Baja California Sur, la prevalencia previamente reportada por alguno de los autores referidos fue mayor que la actual, y únicamente en el Estado de México y en Oaxaca la prevalencia actual fue menor que la reportada por los dos autores.

En el Cuadro 3 se muestra la comparación de prevalencia nacional al nacimiento de hipotiroidismo congénito, en la que se observa el incremento 1.8 veces mayor de 4.1 en 2004 a 7.3× 10,000 recién nacidos vivos en la actualidad.

Cuadro 3. Comparación de la prevalencia al nacimiento de hipotiroidismo congénito, publicada por distintos autores en México*

\begin{tabular}{|l|c|c|c|c|c|}
\hline Autor & Año de publicación & RN analizados & $\begin{array}{c}\text { Duración del } \\
\text { estudio (meses) }\end{array}$ & $\begin{array}{c}\text { Casos } \\
\text { detectados }\end{array}$ & $\begin{array}{c}\text { Tasa de prevalencia, } \\
\text { por 10,000 RN }\end{array}$ \\
\hline Vela-Amieva y col. & 2004 & $1,397,717$ & 12 & 558 & 4.1 \\
\hline Rendón-Macías & 2008 & $2,777,292$ & 48 & 1,286 & 4.3 \\
\hline Presente trabajo & 2018 & $1,267,122^{* *}$ & 17 & 923 & 7.3
\end{tabular}

*Solo se incluyen aquellos estudios que comprendieron todo el país, con cobertura mayor de $85 \%$.

**Sólo incluyó población de la Secretaría de Salud, sin ningún tipo de derechohabiencia.

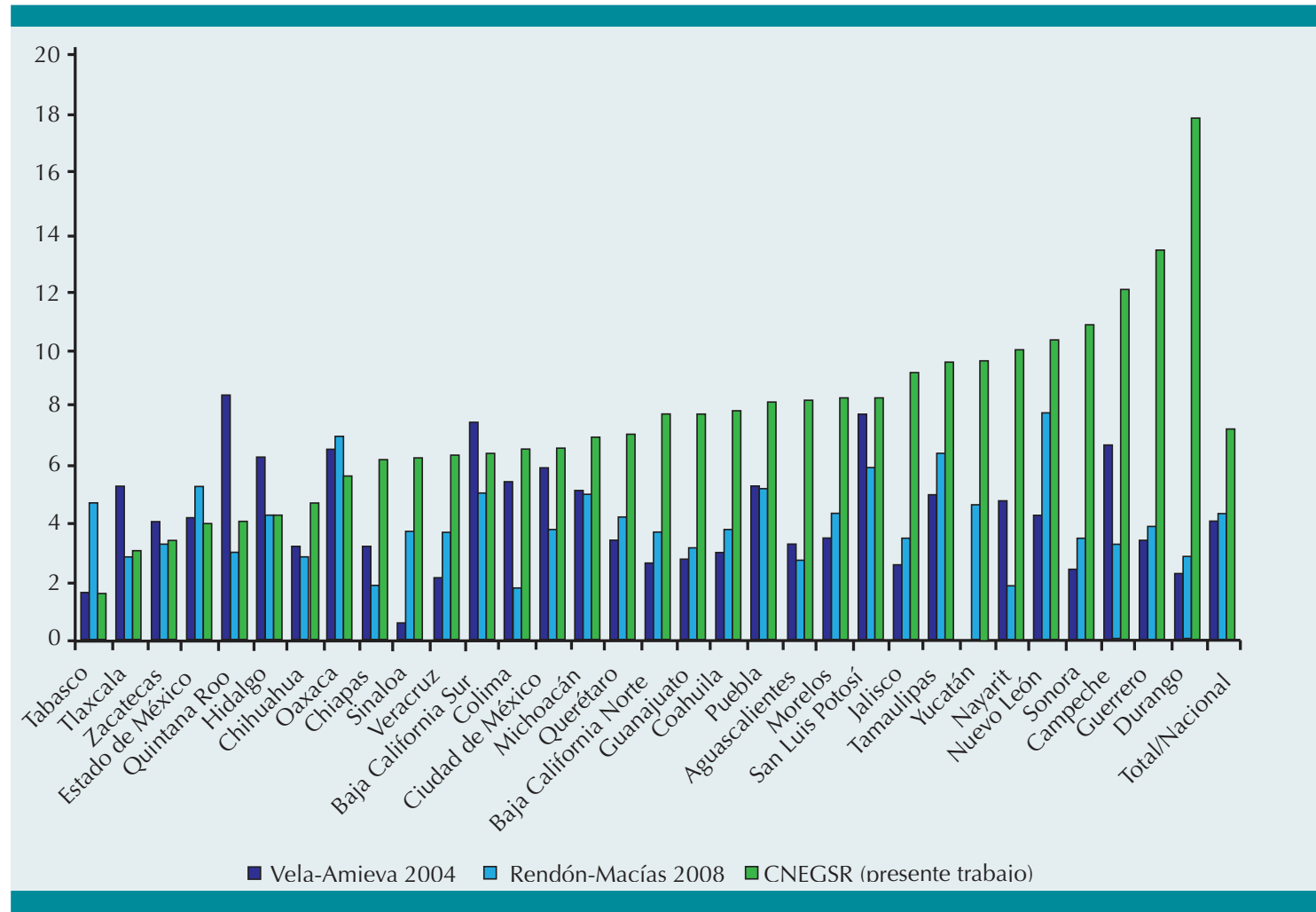

Figura 1. Prevalencias obtenidas de los estados de la República Mexicana. 


\section{DISCUSIÓN}

El presente estudio demuestra que la prevalencia al nacimiento de hipotiroidismo congénito ha aumentado en un periodo de 14 años en México (Cuadro 3, Figura 1). El aumento de la prevalencia al nacimiento de hipotiroidismo congénito es un hecho que se ha observado en muchos países y regiones del mundo y algunos investigadores han encontrado prevalencias de hasta $6.5 \times 10,000$ recién nacidos en los últimos 3 años (Cuadro 4). ${ }^{21-26}$ Estudios internacionales demuestran que los grupos poblacionales que tienen mayor prevalencia de hipotiroidismo congénito son los asiáticos y los hispánicos, entre los que se encuentran clasificadas aquellas personas provenientes de América Latina y los que tienen menores prevalencias son los afrodescendientes. ${ }^{17}$

Las causas del aumento del hipotiroidismo congénito son desconocidas. ${ }^{17}$ Entre las primeras explicaciones se encuentran los cambios en los protocolos de tamiz neonatal en el mundo especialmente la disminución de los valores de corte, $^{24,25,27}$ lo que ha llevado a el aumento de la detección de casos transitorios, ${ }^{17,25,28,29}$ y de casos leves. ${ }^{30}$ Otros autores sostienen que las variaciones en la prevalencia pueden reflejar diferencias en la susceptibilidad genética causada por polimorfismos o por diferentes exposiciones ambientales, por ejemplo: contaminantes que pueden actuar como disruptores endocrinos; ${ }^{31}$ sin embargo, ninguna de estas se ha demostrado. Tampoco hay que olvidar que la asociación del parto pretérmino y del bajo peso al nacimiento con hipotiroidismo congénito, puede reflejar un sobrediagnóstico de la enfermedad, a expensas de casos transitorios. ${ }^{17}$

En este estudio encontramos que hay entidades del país con tasas muy elevadas de hipotiroidismo congénito, destacando Durango (18.0 por 10,000 recién nacidos), Guerrero (13.4 x 10,000 recién nacidos) y Campeche (12.0 por 10,000 recién nacidos) (Cuadro 2), pero hasta el momento no tenemos una explicación de este hecho.

Una limitante del presente trabajo es que no se realizaron en todos los casos los estudios de imagen para conocer el tipo de hipotiroidismo congénito, y definir la etiología del Hipotiroidismo congénito es esencial para saber por qué está aumentando esta enfermedad. ${ }^{32}$

Al igual que otras publicaciones nacionales e internacionales, ${ }^{17,18,19}$ nuestros resultados confirman que el hipotiroidismo congénito es 1.6 veces más frecuente en niñas que en niños.

Cuadro 4. Prevalencias en otras regiones del mundo

\begin{tabular}{|l|c|c|}
\hline País-año & $\begin{array}{c}\text { Prevalencia } \\
\text { por 10,000 RN }\end{array}$ & Referencia \\
\hline Australia, 2002 & 3.5 & Kurinczuk JJ, et al. \\
\hline Quebec, 2011 & 4.0 & Deladoëy J, et al. \\
\hline Italia, 2015 & 5.0 & Olivieri A, et al. \\
\hline Grecia, 2010 & 5.7 & Mengreli C, et al. \\
\hline $\begin{array}{l}\text { Massachussets, } \\
\text { 2011 }\end{array}$ & 6.0 & Mitchell ML, et al. \\
\hline Irlanda, 2018 & $6.5^{*}$ & McGrath \\
\hline
\end{tabular}

*En los últimos 3 años

\section{CONCLUSIÓN}

La prevalencia al nacimiento de hipotiroidismo congénito en la población de recién nacidos en la Secretaría de Salud de México fue de 7.3 x cada 10,000 recién nacidos vivos tamizados, lo que significa que uno de cada 1,373 recién nacidos tiene esta condición; las niñas son las más afectadas. El hipotiroidismo congénito ha aumentado 1.8 veces en los últimos 14 años.

\section{REFERENCIAS}

1. Velázquez A, Loera-Luna A, Aguirre BE, Gamboa S, Vargas $\mathrm{H}$, Robles $\mathrm{C}$. [Neonatal screening for congenital hypothyroidism and phenylketonuria]. Salud Publica Mex. 1994;36(3):249-56. 
2. Norma Técnica Número 321 Para la Prevención del Retraso Mental producido por Hipotiroidismo Congénito. Diario Oficial de la Federación, 22/09/1988, México.

3. Norma Oficial Mexicana NOM-007-SSA2-2016, Para la atención de la mujer durante el embarazo, parto y puerperio, y de la persona recién nacida. Diario Oficial de la Federación, 01/04/2016, México.

4. Loera Luna A, Aguirre BE, Gamboa S, Vargas Flores H, Robles Valdés C, Velázquez Arellano A. Resultados del Programa para la Prevención del Retraso Mental producido por hipotiroidismo congénito Bol Med Hosp Infant Mex 1996; 53(6):259-263.

5. Vela Amieva M, Aguirre BE., Zamudio AM., Gamboa S, von Schmelling $G$, Catalán $A$, et al. Técnica de toma de sangre del cordón umbilical para tamiz neonatal. Acta Pediatr Méx 2000; 21(6): 252-256.

6. Lineamiento Técnico: Tamiz neonatal: Detección y tratamiento oportuno e integral del hipotiroidismo congénito. Centro Nacional de Equidad de Género y Salud Reproductiva. Secretaría de Salud. 1a Ed., 2007.

7. Reglas de Operación del Programa Seguro Médico Siglo XXI, para el ejercicio fiscal 2018. Diario Oficial de la Federación. Acuerdo por el que se emiten las Reglas de Operación del Programa Seguro Médico Siglo XXI, para el ejercicio fiscal 2018. 30 de diciembre de 2017.

8. Rastogi MV, LaFranchi SH. Congenital hypothyroidism. Orphanet Journal of Rare Diseases. 2010; 5:17. doi:10.1186/1750-1172-5-17.

9. Díaz A, Lipman Díaz EG. Hypothyroidism. Pediatr Rev. 2014;35(8):336-47.

10. Léger J, Olivieri A, Donaldson M, et al. European Society for Pediatric Endocrinology Consensus Guidelines on Screening, Diagnosis, and Management of Congenital Hypothyroidism. The Journal of Clinical Endocrinology and Metabolism. 2014;99(2):363-384.

11. Wassner A. J., Brown R. S. Congenital hypothyroidism: Recent advances. Current Opinion in Endocrinology, Diabetes and Obesity. 2015;22(5):407-412.

12. Cherella CE, Wassner AJ. Congenital hypothyroidism: insights into pathogenesis and treatment. International Journal of Pediatric Endocrinology. 2017; 2017:11. doi:10.1186/ s13633-017-0051-0.

13. Castilla-Peón MF. Hipotiroidismo congénito. Bol Med Hosp Infant Mex. 2015;72(2):140-148.

14. Prevención, detección y diagnóstico de hipotiroidismo congénito neonatal en el primer nivel de atención. Guía de Práctica Clínica, Catálogo Maestro: ISSSTE-135/108, México: Secretaría de Salud, 2008.

15. Ford G, LaFranchi SH. Screening for congenital hypothyroidism: a worldwide view of strategies. Best Pract Res Clin Endocrinol Metab. 2014;28(2):175-87.

16. Lorey FW, Cunningham GC. Birth prevalence of primary congenital hypothyroidism by sex and ethnicity. Hum Biol. 1992;64(4):531-8.
17. Hinton $C F$, Harris KB, Borgfeld L, Drummond-Borg M, Eaton $R$, Lorey $F$, et al. Trends in incidence rates of congenital hypothyroidism related to select demographic factors: data from the United States, California, Massachusetts, New York, and Texas. Pediatrics. 2010;125 (suppl 2): S37-S47.

18. Vela-Amieva M, Gamboa-Cardiel S, Pérez-Andrade ME, Ortiz-Cortés J, González-Contreras CR, Ortega-Velázquez V. [Epidemiology of congenital hypothyroidism in Mexico]. Salud Publica Mex. 2004;46(2):141-8.

19. Rendón-Macías ME, Morales-García I, Huerta-Hernández E, Silva-Batalla A, Villasís-Keever MA. Birth prevalence of congenital hypothyroidism in Mexico. Paediatr Perinat Epidemiol. 2008 Sep;22(5):478-85. doi: 10.1111/j.13653016.2008.00955.x.

20. Norma Oficial Mexicana NOM-034-SSA2-2013, Para la prevención y control de los defectos al nacimiento. Diario Oficial de la Federación, 24/06/2014, México.

21. Kurinczuk JJ, Bower C, Lewis B, Byrne G. Congenital hypothyroidism in Western Australia 1981- 1998. J Paediatr Child Health. 2002;38(2):187-191.

22. Deladoëy J, Ruel J, Giguère $Y$, Van Vliet $G$. Is the incidence of congenital hypothyroidism really increasing? A 20-year retrospective population-based study in Québec. J Clin Endocrinol Metab. 2011;96(8):2422-2429.

23. Olivieri A, Fazzini C, Medda E; Italian Study Group for Congenital Hypothyroidism. Multiple factors influencing the incidence of congenital hypothyroidism detected by neonatal screening. Horm Res Paediatr. 2015;83(2):86-93.

24. Mengreli C, Kanaka-Gantenbein C, Girginoudis $P$, et al. Screening for congenital hypothyroidism: thesignificance of threshold limit in falsenegative results. J Clin Endocrinol Metab. 2010;95(9):4283-4290.

25. Mitchell ML, Hsu HW, Sahai I; Massachusetts Pediatric Endocrine Work Group. The increased incidence of congenital hypothyroidism: fact or fancy? Clin Endocrinol (Oxf). 2011;75(6):806-810.

26. McGrath N, Hawkes CP, McDonnell CM, Cody D, O'Connell SM, Mayne PD, et al.Incidence of Congenital Hypothyroidism Over 37 Years in Ireland. Pediatrics. 2018 Sep 21. pii: e20181199. doi: 10.1542/peds.2018-1199.

27. Corbetta C, Weber G, Cortinovis F, Calebiro D, Passoni A, Vigone MC, et. al.A 7-year experience with low blood TSH cutoff levels for neonatal screening reveals an unsuspected frequency of congenital hypothyroidism ( $\mathrm{CH}$ ).Clin Endocrinol (Oxf). 2009;71(5):739-45.

28. Harris KB, Pass KA. Increase in congenital hypothyroidism in New York State and in the United States. Mol Genet Metab. 2007;91(3):268-77.

29. Parks JS, Lin M, Grosse SD, Hinton CF, Drummond-Borg M, Borgfeld $\mathrm{L}$, et al. The impact of transient hypothyroidism on the increasing rate of congenital hypothyroidism in the United States. Pediatrics. 2010;125 Suppl 2: S54-63.

30. Korada SM, Pearce M, Ward Platt MP, Avis E, Turner S, Wastell $\mathrm{H}$, et al. Difficulties in selecting an appropriate 
neonatal thyroid stimulating hormone (TSH) screening threshold. Arch Dis Child. 2010;95(3):169-73.

31. Stoppa-Vaucher S, Van Vliet G, Deladoëy J. Variation by Ethnicity in the Prevalence of Congenital Hypothyroidism Due to Thyroid Dysgenesis. Thyroid. 2011; 21(1):13-18.
32. Albert BB, Cutfield WS, Webster D, Carll J, Derraik JG, Jefferies C, Gunn AJ, Hofman PL. Etiology of increasing incidence of congenital hypothyroidism in New Zealand from 1993-2010. J Clin Endocrinol Metab. 2012;97(9):3155-60. doi: 10.1210/jc.2012-1562. 